\title{
A Remark on Exponential Dynamical Localization in a Long-range Potential
}

\author{
Victor Chulaevsky
}

Department of Mathematics, University of Reims, France

Copyright (c) 2017 by authors, all rights reserved. Authors agree that this article remains permanently open access under the terms of the Creative Commons Attribution License 4.0 International License

\begin{abstract}
Exponential decay of eigenfunctions and of their correlators is shown to occur in two Anderson models on the lattice of arbitrary dimension, with summable decay of infinite-range correlations of the random potential. For the proof, we check the applicability of the Fractional Moment Method.
\end{abstract}

Keywords Anderson Localization, Long-range Potentials

\section{Introduction}

This paper focuses on the localization phenomena in correlated disordered quantum systems on a lattice $\mathbb{Z}^{d}, d \geq 1$. Recall that pioneering results in this general direction, but for non-correlated random potentials on a multi-dimensional lattice, have been obtained by Fröhlich et al. [13, 12].

Shortly after reformulation of the Multi-Scale Analysis in [6], in the framework of random potentials with IID (independent and identically distributed) values on a lattice $\mathbb{Z}^{d}, d \geq 1$, von Dreifus and Klein [7] treated a more challenging case of a stationary Gaussian potentials with a power-law decay of correlations on $\mathbb{Z}^{d}$.

Kirsch et al. [17] considered Anderson model in $\mathbb{R}^{d}$ with an alloy-type potential

$$
V_{x}(\omega)=V_{0}(x)+\sum_{a \in \mathbb{Z}^{d}} \mathfrak{q}_{y}(\omega) \mathfrak{u}(y-a),
$$

where $V_{0}$ is a non-random periodic background potential, random amplitudes $\mathfrak{q}_{y}(\omega)$ (referred to as scatterers' amplitudes) are IID with bounded probability density, and $\mathfrak{u}: \mathbb{R}_{+} \rightarrow \mathbb{R}$ (scatterer, or bump potential) decays polynomially at infinity; in particular, $\mathfrak{u} \in \mathrm{L}^{1}\left(\mathbb{R}^{d}\right)$ (in fact, a faster decay is required; see [17]). Using the Multi-Scale Analysis, they proved Anderson localization near the spectral edges, the number and location of which depends upon $V_{0}$.

Fischer et al. [10, 11] considered an analytically more difficult case of a random Schrödinger operator in $\mathbb{R}^{d}$ with the potential $V(x ; \omega)$ given by a sample of a stationary Gaussian field with continuous argument $x \in \mathbb{R}^{d}$. Under the weakest among their hypotheses on the decay of correlations, viz. a polynomial decay of the function

$$
c(r)=\sup _{|x|=r} \mid \mathbb{E}[V(0 ; \omega) V(x ; \omega] \mid,
$$

they established a similar summable decay of the EFs.

Karsten et al. [16] considered an alloy model with exponentially decaying scatterers. Using the MSA, they proved exponential spectral localization.

Elgart et al. [8] studied the one-dimensional lattice models with compactly supported scatterer function and the common marginal probability density $\rho$ assuming:

- either $\rho \in W^{1,1}(\mathbb{R})$ and $\left\|\rho^{\prime}\right\|_{L^{1}}$ is small enough, or

- $\operatorname{supp} \rho$ is bounded and $\|\rho\|_{\infty}$ is small enough,

and adapted the FMM to such models. In a subsequent paper [9], the authors proved localization via the MSA in any dimension, assuming that the scatterer potential has fixed sign at the boundary of its support.

Kostrykin and Veselić [20] studied the regularity of the IDS in a non-local alloy model with a polynomial decay of the scatterer potential; a Wegner-type estimate they proved is one of the key ingredients of the MSA. Veselić [25] proved a similar estimate in the Gaussian alloy model in $\mathbb{R}^{d}$. See also the work [26] where the two-point eigenvalue correlation measure is studied, from the perspective of Minami estimate and Poisson statistics of the eigenvalues in a correlated environment.

Tautenhahn and Veselić [22], studied an alloy model with Gaussian potential on $\mathbb{Z}^{d}$ having a compactly supported correlation function, thus featuring the IAD (Independence At Distance) property. Aiming to prove Anderson localization in a lattice model via the Fractional Moment Method (FMM; cf. $[3,4])$ they examined the regularity of the single-point probability distribution of the potential at an arbitrary point $x \in \mathbb{Z}^{d}$ conditional on all remaining values $\left\{V(y ; \omega), y \in \mathbb{Z}^{d} \backslash\{y\}\right\}$. A Lipschitz (and even Hölder) continuity of such conditional distributions, uniform in the conditions, would suffice for a successful application of the methods of $[3,4]$. Of course, a conditional Gaussian distribution not supported by a single point is automatically Lipschitz continuous, for it admits a bounded (in fact, analytic) density. The required regularity was established in [22] for a class of IAD Gaussian potentials. The authors also pointed out the existence of alloy potentials with bounded scatterers' amplitudes for which the conditional distributions at hand are not as "regular", from the statistical point of view, as one might think; more precisely, they feature a certain "determinism" in the local interpolation problem. 
Krüger [21] applied the MSA to infinite-range lattice alloy with exponential decay of the scatterer potential and proved spectral and dynamical localization.

In many studied models of correlated media, a slow decay of correlations has been a technical problem, impeding the rate of decay of eigenfunctions and/or their correlators that one was able to prove. It is natural to ask, therefore, to what extent a relatively weak decorrelation really shapes the localization phenomena. The aim of this short note is to shed some additional light on the subject.

The problems arising from correlated nature of the potential are closely related to another important issue discussed in the literature over the last years: non-monotonicity of the eigenvalues (say, in finite domains) due to possible non-signdefiniteness of the scatterer potential. The two principle tools used to address this issue are

(i) subharmonicity arguments, and a matrix version of the Cartan lemma (cf. [5])

(ii) Fourier analysis of the correlated potential (cf., e.g., $[24,16])$.

In the present work, we propose a fairly general sufficient condition for the applicability of the FMM to Gaussian lattice alloy models with infinite-range scatterer potential (hence with a long-range correlation function), possibly sign-indefinite. Since the EV concentration analysis is not required for the FMM in the form of an explicit Wegnertype estimate (albeit the two issues are connected), the logical choice of an efficient tool is (ii), where one inevitably comes to face the local stochasticity (as opposed to local predictability) analysis of the correlated potential at hand. In the Gaussian case, it suffices to apply an old, fairly constructive criterion of minimality (local unpredictability) proved long ago by Kolmogorov [18]. In some cases, it can be validated analytically, and in many more general situations the verification can be assisted by rigorous numerical calculations (not simulations of Monte-Carlo type).

We also obtain some results for a class of long-range alloy models with uniform common marginal distribution of the IID scatterer amplitudes. As mentioned above, in the latter case some conditional distributions can indeed be partially predictable, from the viewpoint of local interpolation, but we show that if

$$
\sum_{y \neq 0}|\mathfrak{u}(y)|<\frac{1}{2} \mathfrak{u}(0),
$$

so that the "central" amplitude $|\mathfrak{u}(0)|$ in the moving average $\sum_{y} \mathfrak{u}(y-x) \mathfrak{q}_{y}(\omega)$ dominates the rest, the conditional distribution of $V_{x}(\cdot)$ given $\left\{V_{y}(\omega), y \neq x\right\}$ is regular enough, being supported on an interval of length uniformly bounded below with a bounded conditional density, so localization still can be established by the FMM.

Comparing this with prior results, it is to be emphasized that in the considered models, the rate of decay of the scatterer potential as such has no visible impact on the rate decay of the EFCs, provided that the $\ell^{1}$-norm is finite and (in the second model) small enough.

\section{Main results}

We show that a solution of the technical problems arising in an attempt to prove Anderson localization for nonlocal alloys, particularly when a fast decay of the eigenfunction correlators (EFCs) is sought, is frustratingly simple for at least two, quite opposite models of local disorder, notably most popular in physics to describe, respectively, (i) unbounded and (ii) bounded random variables: Gaussian distributions and uniform distributions in finite intervals (often referenced to as "finite window" distributions).

The hypotheses of Theorem 2.1 refer to the standard notion of spectral density of a stationary random process on $\mathbb{Z}^{d}$, a function defined on the torus $\mathbb{T}^{d}=\mathbb{R}^{d} /(2 \pi \mathbb{Z})^{d} \cong[-\pi, \pi)^{d}$. See the discussion in the next section and definition, e.g. in [14, Chapter 4] or in [23]. Generally speaking, a stationary process has a spectral measure, but non-predictability (regularity) requires the latter to have a density w.r.t. to the Lebesgue measure on the torus. To illustrate this, recall that the ultimate example of a predictable (singular) Gaussian process is $V_{\omega}(x)=\eta(\omega) \mathrm{e}^{\mathrm{i} \alpha x}$ with a Gaussian $\eta(\cdot)$, in which case the spectral measure is the Dirac's delta-measure at $\alpha$.

Theorem 2.1. Consider a lattice Schrödinger operator $H(\omega)=H_{0}+g V_{x}(\omega), g \neq 0$, with a possibly nonlocal, alloytype stationary Gaussian random potential

$$
V_{x}(\omega)=\sum_{y \in \mathbb{Z}^{d}} \mathfrak{q}_{y}(\omega) \mathfrak{u}(y-x)
$$

where $\left\{\mathfrak{q}_{y}(\cdot), y \in \mathbb{Z}^{d}\right\}$ are IID Gaussian random variables with zero mean and nonzero variance, and $\mathfrak{u} \in \ell^{1}\left(\mathbb{Z}^{d}\right)$ with $\|\mathfrak{u}\| \neq 0$. Assume that the stationary process $V_{\bullet}$ on $\mathbb{Z}^{d}$ admits a spectral density $f: \mathbb{T}^{d} \rightarrow \mathbb{R}_{+}$obeying

$$
\int_{\mathbb{T}^{d}} \frac{d \lambda}{f(\lambda)}<+\infty
$$

Then $H(\omega)$ features exponential dynamical and spectral localization:

- in the entire range of energies, $\mathbb{R}$, provided $|g|$ is large enough;

- for any $|g| \neq 0$ on a half-line $(-\infty,-A]$ with $A>0$ large enough.

In both cases localization can be established by the Fractional Method Moment.

In the case of the random Schrödinger operator in a Euclidean space, also with a Gaussian alloy-type potential and the scatterer's potential $\mathfrak{u}(\cdot)$ with non-compact support, a simple reference to the work by Aizenman et al. [2] would be insufficient, for the authors of [2] assume:

(i) the IAD of the random potential,

(ii) uniform (in $[0,1]$ ) marginal probability distribution.

A direct inspection of the proofs in [2, Section 3.2] suggests that (ii) is a minor issue, from the perspective of adaptation to the nonlocal alloy models in a Euclidean space. Besides, the second model, considered in Theorem 2.2, has a compactly supported smooth marginal probability distribution, but adaptation to the case of infinite-range correlations cannot be claimed or dismissed without a thorough technical analysis. We plan to address this issue in a forthcoming paper.

Theorem 2.2. Consider the random lattice Schrödinger operator $H_{\varepsilon}(\omega)=\varepsilon H_{0}+V(x ; \omega), \varepsilon>0$. Assume that

$$
V(x, \omega)=X_{x}(\omega)+\sum_{y \neq x} X_{y}(\omega) \mathfrak{u}(y-x)
$$


where $X_{y}$ are IID random variables with common distribution $\operatorname{Unif}([0,1])$, and

$$
\delta:=\sum_{y \neq x}|\mathfrak{u}(y-x)|<\frac{1}{2} .
$$

Then for sufficiently small $\varepsilon, H_{\mathcal{E}}(\omega)$ features exponential strong dynamical and spectral localization, which can be established via the Fractional Moments Method.

\section{Non-deterministic (minimal) Gaus- sian potentials. Proof of Theorem 2.1}

The present paper focuses on the FMM approach to the proof of localization under a long-range correlated potential. Recall, therefore, that this approach was originally developed by Aizenman and Molchanov [3] and later significantly generalized in a series of subsequent works, starting with [1,4], often bearing a distinctive mark of Michael Aizenman's enthusiasm.

The dependence at distance gives rise to unpleasant technical problems in the MSA, where the usual way to get through (particularly, in a continuous configuration space where this is inevitable, unless the potential is a decorrelated random field with non-measurable samples) is to assume Independence At Distance (IAD) property, i.e., absence of any dependence at some finite distance. On the contrary, the Moment Analysis is to a large extent free of this limitation: the IAD can be safely replaced by regularity of conditional distributions induced on one or two points, given the values of the potential at all remaining points. This feature of the Moment Analysis has been emphasized already by Aizenman and Molchanov (cf. [3, Eqns. (2.7), (2.14)-(2.18)]) and again in $[4$, Section 1.2$]$.

Hundertmark [15] proposed a very simple and elegant reformulation of the FMM, in the case of strong disorder on the lattice. The starting point for his analysis is a re-summation of the formal perturbation series for the inverse $\left(H_{0}+V-\right.$ $E)^{-1}$ via "elimination of loops" in the usual graphic expansion (alas, divergent in vicinity of the spectrum). The resulting series is not only convergent, but reduces to a finite sum (over self-avoiding walks) on any finite graph. As argued in $[1,4]$ a uniform control of EFCs in finite subgraphs of an infinite graph implies decay of the EFCs on the infinite graph, with the help of Fatou's lemma for converging (spectral) measures.

The key to the fast decay of the eigenfunction correlators, starting with the decay analysis of the Green functions, is the Hölder regularity of the single-point conditional distribution of the random potential at $x \in \mathbb{Z}^{d}$, given all the remaining values on $\mathbb{Z}^{d} \backslash\{x\}$. In [15] the latter appear in the so-called self-energy entering one-dimensional integrals over the conditional measure. Naturally, in the Gaussian case the conditional measure is either supported by a single point or analytic, with rapidly decaying tail probabilities.

For technical details we refer the to the original works [3, $1,4,15]$.

\subsection{Interpolation and criteria of pointwise un- predictability}

To avoid a terminological confusion, it is to be stressed that in many books, lecture courses and research papers, by unpredictability of a random process on $\mathbb{Z}^{1}$ one means nondegeneracy of the distribution of $X_{S}(\cdot)$ conditional on all previous values $X_{t}(\omega), t<s$. Needless to say, the irreversible temporal evolution of real world makes the prediction problem quite special and popular, and to realise that, one does not even have to spend long hours in a large library; a quick Google search would bring (as the experience shows) a lot more papers on prediction (also called extrapolation) than on the local, even one-point interpolation problem in the sense we need in this paper. Also, the most often quoted, celebrated criteria of non-determinicity of a random process concern the prediction problem (see in particular the original paper by Kolmogorov [19] and a more general presentation in the monograph [14]). For these reasons and for the reader's convenience, below are recalled some fundamental results on interpolation obtained almost a century ago.

An English translation of the works $[19,18]$ can be found in A. N. Kolmogorov's Selecta [23] (cf. papers no. 27 and 28).

Kolmogorov [19, 18] considered and solved the singlepoint interpolation problem for a stationary random process $X_{t}(\omega)$ with discrete time $\mathbb{Z}$; in particular, he obtained a criterion of exact solvability of the single-point interpolation problem, and calculated the exact value of the variance of the minimal interpolation error whenever the latter has no exact solution. Later on, A. M. Yaglom [27] solved in a similar way a more general problem of interpolation on a finite number of points $t_{1}, \ldots, t_{n}, n>1$. We quote below the statement of [27, Theorem 2] on the interpolation problem and interpolation error, particularly taking into account that [27] may be unavailable in English or difficult to access for some readers.

The statement of [27, Theorem 2] is reproduced almost verbatim, so it is worthwhile mentioning that absolute continuity of the spectral function $F(\lambda)$, with derivative $F^{\prime}(\lambda)$ well-defined Lebesgue-a.e. on $[-\pi, \pi)$, is implicitly assumed below. The lattice $\mathbb{Z}$ is partitioned as $K \sqcup K^{\prime}$, where $K^{\prime}$ is a finite set on which the (missing) values $X_{s}, s \in K^{\prime}$, are to be recovered with the best available accuracy, by linear interpolation of the known values $X_{r}, r \in K$, i.e., by the linear estimators

$$
L_{n}=\sum_{i=1}^{n} a_{i} X_{t_{i}}, t_{i} \in K, i=1, \ldots, n .
$$

Further, one considers the Hilbert space spanned by the random variables $X_{t}(\omega), t \in \mathbb{Z}$ assumed to have zero mean and finite variance; the scalar product is given by $\langle X, Y\rangle=$ $\mathbb{E}[X Y]$. The accuracy of the (linear) interpolation is measured by

$$
\sigma^{2}\left(s, L_{n}\right):=\mathbb{E}\left[\left|X_{s}-L_{n}\right|^{2}\right] .
$$

The solvability and accuracy of the interpolation problem are studied in terms of the quantities

$$
\sigma^{2}(s, K)=\inf _{L_{n}} \sigma^{2}\left(s, L_{n}\right), s \in K^{\prime} .
$$

The spectral function $F=F-X$ and its derivative (whenever it exists), $f_{X}=F_{X}^{\prime}$, figuring in the statements and arguments below, are related to the covariance function $C(t)=$ $\mathbb{E}\left[X_{0} X_{t}\right]$ of a stationary process $X_{\bullet}$ with discrete time $\mathbb{Z}^{d}$ 
in the following way. Aiming to represent infinite samples $\left(X_{t}, t \in \mathbb{Z}^{d}\right)$ as Fourier-transforms of samples of a random measure (called random spectral measure of the process $X_{\bullet}$ ) on the torus $\mathbb{T}^{d}$, one has first to construct such a measure; a procedure requiring some time and efforts, cf., e.g., [14, Chapter 4] or [19]. An important feature of the (random) spectral measure $d Z_{\omega}(\lambda)$ is its orthogonality property: for some non-random Stieltjes measure $d F(\lambda)$ and any two Borel subsets $A, B \subset \mathbb{T}^{d}$, one has

$\mathbb{E}\left[\int_{\mathbb{T}^{d}} \mathbf{1}_{A}(\lambda) d Z_{\omega}(\lambda) \cdot \int_{\mathbb{T}^{d}} \mathbf{1}_{B}(\lambda) d Z_{\omega}(\lambda)\right]=\int_{\mathbb{T}^{d}} \mathbf{1}_{A \cap B}(\lambda) d F(\lambda)$.

of the random spectral measure $d Z_{\omega}(\lambda)$, or spectral function of the process $X_{\bullet}$. Whenever $F$ is a.e. differentiable, its derivative is called spectral density.

It is worth noticing that the construction of the spectral mesures, quite formal, relies only upon the covariance function and in general bears no information pertaining to the marginal probability distribution of $X_{t}$. For example, all IID (hence delta-correlated) processes are alike from this "linear" perspective, whether $X_{t}$ takes a finite number of values or has analytic probability density. It goes without saying that this is a great relief in many practical applications, but also significantly limits the theoretical value of the linear theory of general stationary processes, without additional specific hypotheses on their nature. In particular, this sets apart Gaussian stationary processes.

Below we set $f_{X}=F_{X}^{\prime}$, to have the same notations in this and the next subsection.

Theorem 3.1 ([27, Theorem 2]). Suppose that the spectral function $F(\lambda)$ of a stationary sequence $\left\{X_{t}\right\}$ is such that there exists a set of complex numbers $\alpha=\left(\alpha_{1}, \ldots, \alpha_{n}\right)$ for which the integral

$$
R(\alpha)=\int_{-\pi}^{\pi} \frac{\left|1+\sum_{k=1}^{n} \alpha_{k} \mathrm{e}^{\mathrm{i}\left(t_{k}-s\right) \lambda}\right|^{2}}{f_{X}(\lambda)} d \lambda
$$

converges, then the quantity $\sigma^{2}(s, K)$, with a finite set $K^{\prime}=$ $\left\{s, t_{1}, \ldots, t_{n}\right\}$, is given by

$$
\sigma^{2}(s, K)=\frac{4 \pi^{2}}{\min _{\alpha} R(\alpha)}
$$

If the integral (3.1) diverges for any choice of $\alpha$, then one has $\sigma^{2}(s, K)=0$.

Clearly, a simpler, albeit less precise, sufficient condition for local unpredictability (on a finite set) is given by

$$
\mathscr{J}\left(f_{X}\right)=\int_{-\pi}^{\pi} \frac{1}{f_{X}(\lambda)} d \lambda<+\infty
$$

Thus

$$
\sigma^{2}(s, K) \geq \frac{4 \pi^{2}}{R(\mathbf{0})}=\frac{4 \pi^{2}}{\mathscr{J}\left(f_{X}\right)} .
$$

On the other hand, it is equally clear that the hypothesis of convergence of the integral (3.3) is significantly weaker than the strict positivity of the spectral density:

$$
\inf _{\lambda \in[-\pi, \pi]} F^{\prime}(\lambda) \geq c>0 .
$$

In the particular case where the interpolation set contains a single point, $K^{\prime}=\{s\}$, the above sufficient condition becomes a criterion. Here we return to the original result by Kolmogorov.
In order to keep the original formulation of [18, Theorem 24], call, following [18], a stationary random sequence $X: \mathbb{Z} \times \Omega \rightarrow \mathbb{R}$ minimal iff the probability distribution of the random variable $X_{0}(\omega)$ conditional on the sigma-algebra $\mathfrak{F}_{\neq 0}$ generated by $\left\{X_{t}(\cdot), t \neq 0\right\}$ is non-degenerate, i.e., not supported by a single point. In other words, the interpolation problem for the value $X_{0}(\omega)$ (and by stationarity, for any value $X_{s}(\omega)$ ) does not admit an exact solution. A more constructive criterion of pointwise non-determinicity of $X_{\bullet}(\omega)$ is strict positivity of the conditional variance

$$
\sigma_{0}^{2}=\mathbb{E}\left[\left(X_{0}(\omega)-\mathbb{E}\left[X_{0}(\omega) \mid \mathfrak{F}_{\neq 0}\right]\right)^{2} \mid \mathfrak{F}_{\neq 0}\right] .
$$

Theorem 3.2 (Cf. [18, Theorem 24]). A stationary random sequence $\left(X_{t}(\omega)\right)_{t \in \mathbb{Z}}$ is minimal iff its spectral density $f_{X}$ : $\lambda \mapsto \mathbb{R}_{+}, \lambda \in[-\pi, \pi)$, is strictly positive Lebesgue-a.e. on $[-\pi, \pi)$ and one has

$$
\mathscr{J}\left(f_{X}\right):=\int_{-\pi}^{\pi} \frac{d \lambda}{f_{X}(\lambda)}<\infty .
$$

Under these conditions, the single-point interpolation variance is given by

$$
\sigma^{2}\left(X_{\bullet}\right)=\frac{4 \pi^{2}}{\mathscr{J}\left(f_{X}\right)}>0 .
$$

From the perspective of applications to the complete, FMM-style single-point decoupling of the correlated potential of alloy type with an infinite-range scatterer function, the strict positivity of the conditional variance per se is certainly insufficient in general, for it provides no information on the regularity of the respective conditional distribution. The Gaussian case, however, is quite special, since (i) a conditional distribution for a Gaussian measure is always Gaussian (possibly degenerate), and (ii) any Gaussian measure on $\mathbb{R}$ with nonzero variance is automatically Lipschitz continuous (even analytic).

\subsection{Multi-dimensional criterion of minimality}

Kolmogorov's criterion [18, Theorem 24] extends easily to the multidimensional lattices $\mathbb{Z}^{d}, d>1$. This probably explains the fact that it is difficult to make a direct reference to a research paper where the multidimensional case has been treated: any mathematical journal would certainly qualify such an extension as a mere exercise, worth perhaps including it in a textbook.

Below we sketch necessary calculations to adapt the formula for the interpolation variance to $d>1$, but for brevity, state and prove only the sufficiency of the condition (3.6), although it actually remains a criterion, as in the one-dimensional case. The necessity is proved along the lines of [18].

Theorem 3.3. Suppose that a stationary random sequence $\left(X_{t}(\omega)_{t \in \mathbb{Z}^{d}}\right.$ admits a spectral density $f_{X}: \lambda \mapsto \mathbb{R}_{+}, \lambda \in \mathbb{T}^{d}$ which is strictly positive Lebesgue-a.e. on $\mathbb{T}^{d}$ and obeys

$$
\mathscr{J}\left(f_{X}\right):=\int_{\mathbb{T}^{d}} \frac{d \lambda}{f_{X}(\lambda)}<\infty .
$$

Then $X_{\bullet}$ is pointwise non-deterministic, with the single-point interpolation variance

$$
\sigma^{2}\left(X_{\bullet}\right)=\frac{(2 \pi)^{2 d}}{\mathscr{J}\left(f_{X}\right)}>0 .
$$


Proof. Assume that (3.6) holds true. Then the weighted Hilbert space $\mathrm{L}^{2}\left(\mathbb{T}^{d}, f_{X} d \lambda\right)$ is isometrically equivalent to the standard one, $\mathrm{L}^{2}\left(\mathbb{T}^{d}\right) \equiv \mathrm{L}^{2}\left(\mathbb{T}^{d}, d \lambda\right)$, and the equivalence

$$
U: \mathrm{L}^{2}\left(\mathbb{T}^{d}\right) \rightarrow \mathrm{L}^{2}\left(\mathbb{T}^{d}, f_{X} d \lambda\right)
$$

is established by $\left(U^{-1} \phi(\lambda)\right):=\sqrt{f_{X}(\lambda)} \phi(\lambda)$.

Further, denote by $\Phi$ the isometry between $\mathscr{H}^{(X)}$ and its spectral representation, so $\Phi\left(X_{n}\right)=\mathrm{e}^{\mathrm{i} n \cdot(\cdot)}: \mathbb{T}^{d} \rightarrow \mathbb{C}$. The subspace $\mathscr{H}_{\neq 0}^{(X)}$ is spanned, in the original representation, by the random variables $X_{t}(\omega), t \in \mathbb{Z}^{d} \backslash\{0\}$, and in the spectral representations by the exponential functions $\left\{\mathrm{e}^{\mathrm{i} n \cdot \lambda}, n \neq 0\right\}$. Consequently, its orthogonal complement has dimension 1 . Note that

$$
\|1 / f\|^{2}=\int_{\mathbb{T}^{d}} \frac{1}{f^{2}(\lambda)} f(\lambda) d \lambda=\mathscr{J}\left(f_{X}\right)<\infty
$$

so $1 / f$ is in the Hilbert space, and

$$
\forall n \neq 0 \quad \int_{\mathbb{T}^{d}} \frac{1}{f(\lambda)} \mathrm{e}^{-\mathrm{i} n \cdot \lambda} f(\lambda) d \lambda=0 .
$$

Thus $\Phi\left(\mathscr{H}_{\neq 0}\right)$ is spanned by $\varphi:=1 / f$. Now decompose $X_{0}=Y+Z$, with $Z \in \mathscr{H}_{\neq 0}$ and $Y \in \mathscr{H}_{0}$, hence $Y=c \varphi=\frac{c}{f}$. Let $\varphi_{Y}=\Phi(Y), \varphi_{Z}=\Phi(Z)$. By construction $\Phi\left(X_{0}\right)=\mathbf{1}=$ $\varphi_{Y}+\varphi_{Z}$. It follows from the orthogonality of $Y$ and $Z$ that

$$
\begin{aligned}
\|Y\|^{2} & =(Y, Y)=(Y, Y+Z)=\left(\varphi_{Y}, \mathbf{1}\right) \\
& =\int_{\mathbb{T}^{d}} \frac{c}{f_{X}(\lambda)} \cdot 1 \cdot f_{X}(\lambda) d \lambda=c(2 \pi)^{d} .
\end{aligned}
$$

Hence

$$
\begin{gathered}
c=\frac{\|Y\|^{2}}{(2 \pi)^{d}}, \varphi_{Y}(\lambda)=\frac{c}{f(\lambda)}=\frac{\|Y\|^{2}}{(2 \pi)^{d} f(\lambda)}, \\
\|Y\|^{2}=\left\|\varphi_{Y}\right\|^{2}=\frac{\|Y\|^{4}}{(2 \pi)^{2 d}}\|f(\lambda)\|^{2}=\frac{\|Y\|^{4}}{(2 \pi)^{2 d}} \mathscr{J}\left(f_{X}\right) .
\end{gathered}
$$

Solving for $\|Y\|^{2}$, we obtain the required identity

$$
\|Y\|^{2}=\frac{(2 \pi)^{2 d}}{\mathscr{J}\left(f_{X}\right)}
$$

Thus the stated condition is sufficient and implies the interpolation variance (3.8).

We conclude that for the Gaussian alloy potentials satisfying the condition (3.3) the FMM is applicable, thus proving strong dynamical localization with exponentially decaying EFCs - under one of the two usual assumptions, in essence having the same meaning: weak concentration of the Density of States measure in the investigated energy interval. One of the assumptions is strong disorder (or small hopping amplitude in front of the Laplacian); the other restricts the localization analysis to sufficiently low ("extreme") energies (in the Gaussian case).

In the next section we treat another popular probability distribution of the scatterers' amplitudes, often used in physics to model probability distributions with compact support: a uniform distribution in a bounded interval.

\section{Uniform distribution of scatterers' amplitudes. Proof of Theorem 2.2}

Lemma 4.1. Let $V(x, \omega)=X_{x}(\omega)+\sum_{y \neq x} X_{y}(\omega) \mathfrak{u}(y-x)$, with IID $X_{y} \sim \operatorname{Unif}([0,1])$. If

$$
\widehat{\varepsilon}:=\sum_{y \neq 0}|\mathfrak{u}(y)|<\frac{1-\delta}{2}, \text { for some } \delta>0,
$$

then the conditional probability distribution $\mathbb{P}\left\{\cdot \mid \mathfrak{F}_{\neq x}\right\}$ admits a bounded density $\rho_{x}\left(\cdot \mid \mathfrak{F}_{\neq x}\right)$ with $\left.\left\|\rho_{x}\left(\cdot \mid \mathfrak{F}_{\neq x}\right)\right\|\right|_{\infty} \leq$ $\delta^{-1}$.

Proof. It suffices to consider a finite subgraph of the lattice, $\mathscr{Z}$, labeling the random variables $X_{\bullet}$; in the general case, the uniform upper bound can be obtained by standard approximation arguments (Fatou's lemma; cf. [1, 4]), once a bound uniform in the size of the graph is obtained for all arbitrarily large but finite graphs.

The probability measure of the random vector $\mathbf{X}=\mathbf{X}_{\mathscr{Z}}=$ $\left(X_{x}, x \in \mathscr{Z}\right)$ is the Lebesgue measure on the unit cube $[0,1]^{\mathscr{Z}}$. The random vector $\mathbf{V}=\mathbf{V}_{\mathscr{Z}}=(V(x), x \in \mathscr{Z})$ is obtained by an invertible linear transformation of $\mathbf{X}$ (with $\widehat{\varepsilon}<1$, the inverse is given by the Neumann series), hence with constant Jacobian. The conditional distribution $\mathbb{P}\left\{\cdot \mid \mathfrak{F}_{\neq x}\right\}$ is supported by an interval $J_{x}\left(\mathbf{X}_{x}^{\perp}\right)$ of an affine real line (since the dimension is preserved by a linear isomorphism) $\mathscr{L}_{x}\left(\mathbf{X}_{x}^{\perp}\right) \subset$ $\mathbb{R}^{\mathscr{Z}}$; possibly $J_{x}\left(\mathbf{X}_{x}^{\perp}\right)=\{\lambda\}$ for some point $\lambda$.

A line in $\mathbb{R}^{\mathscr{Z}}$ may of course have an empty intersection with the compact support of the joint measure. More importantly, short intersection intervals $J$ give rise to large values of the conditional density, viz. $|J|^{-1} \mathbf{1}_{J}(\cdot)$.

The role of the quantity $\widehat{\varepsilon}$ becomes clear now: whenever $1-2 \widehat{\varepsilon}=\delta>0$, the support of the conditional measure is an interval of positive length, lower bounded by $\delta>0$. This is readily seen from the fact that for any values of $X_{y} \in[0,1]$, $y \in \mathscr{Z} \backslash\{x\}$, each value $V_{x} \in I_{\widehat{\mathcal{E}}}$ is admissible, for it suffices to set

$$
X_{x}:=V_{x}-\sum_{y \neq x} \mathfrak{u}(y-x) X_{y}
$$

noting that $X_{x} \in[0,1]$, thus in the support of the marginal measure, due to (4.1):

$X_{x} \geq \widehat{\mathcal{\varepsilon}}-\sum_{y \neq x}|\mathfrak{u}(y-x)| X_{y} \geq \widehat{\varepsilon}-\sum_{y \neq x}|\mathfrak{u}(y-x)| \cdot 1>0$,
$X_{x} \leq(1-\widehat{\varepsilon})+\sum_{y \neq x}|\mathfrak{u}(y-x)| X_{y} \geq 1-\widehat{\varepsilon}+\sum_{y \neq x}|\mathfrak{u}(y-x)| \cdot 1<1$.

Once $\left|J_{x}\left(\mathbf{X}_{x}^{\perp}\right)\right| \geq \delta$, the conditional measure is the normalized restriction of the ambient Lebesgue measure in the cube $[0,1]^{\mathscr{Z}}$ on an interval of length $\ell \geq \delta$, hence it has constant density on its support, equal to $\ell^{-1} \leq \delta^{-1}$.

An immediate consequence is applicability of the FMM under the hypotheses of Lemma 4.1. The requirement for $\varepsilon$ in $\varepsilon H_{0}+V$ to be small enough is equivalent to the strong disorder condition.

\section{REFERENCES}

[1] Aizenman, M. Localization at weak disorder: Some elementary bounds. Rev. Math. Phys., Vol.6, 1163-1182, 1994. 
[2] Aizenman, M., Elgart, A., Naboko, S., Schenker, J., Stolz, G. Moment analysis for localization in random Schrödinger operators. Invent. Math., Vol.163, 343-413, 2006.

[3] Aizenman, M., Molchanov, S.A. Localization at large disorder and at extreme energies: an elementary derivation. Commun. Math. Phys., Vol.157, 245-278, 1993.

[4] Aizenman, M., Shenker, J.H., Fridrich, R.M., Hundertmark, D. Finite-volume fractional-moment criteria for Anderson localization. Commun. Math. Phys., Vol.224, 219-253, 2001.

[5] Bourgain, J. An approach to Wegner's estimate using subharmonicity. J. Stath. Phys., Vol.134, 969-978, 2009.

[6] von Dreifus, H., Klein, A. A new proof of localization in the Anderson tight binding model. Commun. Math. Phys., Vol.124, 285-299, 1989.

[7] von Dreifus, H., Klein, A. Localization for random Schrödinger operators with correlated potentials. Commun. Math. Phys., Vol.140, 133-147, 1991.

[8] Elgart, A., Tautenhahn, M., Veselić, I. Localization via fractional moments for models on $\mathbf{Z}$ with single-site potentials of finite support. J. Phys. A, Vol.43, 474,021, 2010.

[9] Elgart, A., Tautenhahn, M., Veselić, I. Anderson localization for a class of models with a sign-indefinite single-site potential via fractional moment method. Annales Henri Poincaré, Vol.12, 1571-1599, 2011.

[10] Fischer, W., Hupfer, T., Leschke, H., Müller, P. Existence of the density of states for multi-dimensional continuum Schrödinger operators with Gaussian random potentials. Commun. Math. Phys., Vol.190, 133-141, 1997.

[11] Fischer, W., Leschke, H., Müller, P. Spectral localization by Gaussian random potentials in multi-dimensional continuous space. J. Stat. Phys., Vol.101, 935-985, 2000.

[12] Fröhlich, J., Martinelli, F., Scoppola, E., Spencer, T. Constructive proof of localization in the Anderson tight binding model. Commun. Math. Phys., Vol.101, 21-46, 1985.

[13] Fröhlich, J., Spencer, T. Absence of diffusion in the Anderson tight binding model for large disorder or low energy. Commun. Math. Phys., Vol.88, 151-184, 1983.

[14] Gikhman, I., Skorohod, A. The theory of stochastic processes I. Classics in Mathematics. Springer-Verlag, 2004.

[15] Hundertmark, D. A short introduction to Anderson localization. In: Anal. Stoch. Growth Int. Models, pp. 194-218. Oxford University Press, Oxford, UK, 2008.

[16] Karsten, L., Peyerimhoff, N., Tautenhahn, M., Veselić, I. Wegner estimate and localization for alloy-type models with sign-changing exponentially decaying single-site potentials. Rev. Math. Phys., Vol.27, 1550007, 2015.

[17] Kirsch, W., Stollmann, P., Stolz, G. Anderson localization for random Schrödinger operators with long range interactions. Commun. Math. Phys., Vol.195, 495-507, 1998.

[18] Kolmogorov, A.N. Interpolation and extrapolation of stationary random sequances. Izvestia Acad. Sci. USSR, Vol.5, 314,1941 .

[19] Kolmogorov, A.N. Staionary sequences in a Hilbert space. Bull. MGU (Moscow State University), Vol.2, 1-40, 1941.

[20] Kostrykin, V., Veselić, I. On the Lipschitz continuity of the integrated density of states for sign-indefinite potentials. Math. Z., Vol.252, 367-392, 2006.
[21] Krüger, H. Localization for random operators with nonmonotone potentials with exponentially decaying correlations. Ann. Henri Poincaré, Vol.13, 543-598, 2012.

[22] Tautenhahn, M., Veselić, I. A note on regularity for discrete alloy-type models. Technische Universität Chemnitz, Preprintreihe der Fakultät für Mathematik, preprint 2010-6, ISSN 1614-8835, 2010.

[23] Tikhomirov, V.M. (ed.) Selected works of A. N. Kolmogorov. Volume II. Probability theory and mathematical statistics. Mathematics and its applications (Soviet Series). Springer, 1992.

[24] Veselić, I. Wegner estimates for sign-changing single site potentials. Math. Phys. Anal. Geom., Vol.13, 299-313, 2010.

[25] Veselić, I. Lipschitz-continuity of the integrated density of states for Gaussian random potentials. Lett. Math. Phys., Vol.97, 25-27 (2011)

[26] Veselić, I. Minamis estimate: Beyond rank one perturbation and monotonicity. Ann. Henri Poincaré, Vol.15, 737-754, 2014.

[27] Yaglom, A. On problems about linear interpolation of stationary random sequences and processes. Uspekhi Mat. Nauk, Vol.4, 173-178, 1949. 